\title{
REDESCUBRIMIENTO DE LA CHINCHILLA ANDINA (CHINCHILLA CHINCHILLA) EN LA RESERVA NACIONAL LOS FLAMENCOS. NUEVO REGISTRO DE CHINCHILLA ANDINA EN LA REGIÓN DE ANTOFAGASTA, CHILE
}

\author{
Hèctor Gallardo ${ }^{1}$, Roberto $\mathrm{Cruz}^{2}$, Alejandro $\mathrm{Cruz}^{3}$, César Romero ${ }^{4}$ y René Novoa ${ }^{5}$ \\ Corporación Nacional Forestal 'hector.gallardo@conaf.cl; ${ }^{2}$ roberto.cruz@conaf.cl; ${ }^{3}$ alejandro.cruz@conaf.cl; \\ ${ }^{4}$ spatacama@yahoo.com; ${ }^{5}$ rene.novoa@conaf.cl
}

\section{RESUMEN}

Se reporta un nuevo hallazgo de una colonia de chinchilla andina para la segunda región de Chile, en el sector 1 de la Reserva Nacional Los Flamencos, donde se desconocía su existencia y se estimaba una baja probabilidad de presencia. El registro fue efectuado en noviembre de 2020 mediante cámaras trampa durante un estudio de la diversidad de meso y macrofauna en dicho sector. Este descubrimiento reciente aporta nuevos datos de su distribución, hábitat y testifica su reaparición en la cordillera de San Pedro de Atacama. En consecuencia, se reafirma la gran importancia de las áreas protegidas en la conservación de la fauna silvestre y se sugiere estudiar la presencia de la especie en Argentina cerca de este lugar, el cual forma parte de un trifinio.

Palabras clave: chinchilla de cola corta, distribución, Salar de Tara y Aguas Caliente I, San Pedro de Atacama.

\section{ABSTRACT}

Rediscovery of the Short-tailed Chinchilla (Chinchilla chinchilla) in Los Flamencos National Reserve. New record of Short-tailed Chinchilla in the Antofagasta Region, Chile. A new finding of a Short-tailed Chinchilla colony is reported for the Second Region of Chile, in sector 1 of Los Flamencos National Reserve, where its existence was unknown, and a low probability of presence was estimated. The record was carried out in November 2020 using camera traps during a study of the diversity of meso- and macrofauna in this sector. This recent discovery provides new data on its distribution, habitat and testifies to its reappearance in the San Pedro de Atacama mountain range. Consequently, the great importance of protected areas in wildlife conservation is reaffirmed, and it is suggested to study the presence of the species in Argentina near this place, which is part of a tri-border area.

Keywords: distribution, Salar de Tara y Aguas Calientes I, San Pedro de Atacama, Short-tailed Chinchilla.

\section{INTRODUCCIÓN}

Tras haber sido considerada extinta en estado silvestre en la década de los 60 por causa de la industria peletera (Jiménez 1996), la chinchilla de cola corta o andina Chinchilla chinchilla (Lichtenstein, 1830) se ha ido redescubriendo en los últimos años, esencialmente, en puntos muy específicos y aislados del norte de Chile (Valladares et al. 2014a; Valladares et al. 2018) en las regiones de Antofagasta (Valladares et al. 2012) y Atacama (Lagos et al. 2012; Navarro et al. 2019). De hecho, en los países colindantes la situación es mucho más pobre. Mientras que en Perú se considera potencialmente extinta (Pacheco et al. 2021), en Argentina solo se obtuvieron registros indirectos encontrados en egagrópilas (Ortiz et al. 2010). Sin embargo, Bolivia recientemente registró sus primeros avistamientos de chinchilla andina en la zona 
suroeste del país, concretamente en la Reserva Nacional de Fauna Andina Eduardo Avaroa (REA), cerca de la frontera con Chile (Delgado et al. 2018) y en puntos más al norte alrededor del Salar de Uyuni (Copa et al. 2014).

Por otra parte, si nos centramos en los puntos de presencia chilenos, los registros más abundantes de dicho roedor se encuentran en el sur de la región de Antofagasta, en el Parque Nacional Llullaillaco (Riquelme et al. 2015) y los más al norte, en la comuna de San Pedro de Atacama, en El Laco (Spotorno et al. 2004) y en el extremo sureste del Salar de Atacama (Salinas et al. 2019). En su punto intermedio de la cordillera de los Andes, en la Comuna de San Pedro de Atacama, se tienen registros en las proximidades del Salar de Atacama (Salinas et al. 2019) y existen avistamientos históricos cerca del volcán Lickancabur (Rudolph 1955).

No obstante, estas colonias aisladas que poco a poco se van redescubriendo siguen estando bajo amenazas, siendo las más graves la explotación minera (Valladares et al. 2014b), la fragmentación de sus poblaciones (Valladares et al. 2012) y, posiblemente aún, su caza ilegal (Mella et al. 2002). Si bien se van recopilando datos del estado y ecología de la especie gracias a los descubrimientos de nuevas colonias, como mapas de distribución potencial (Deane 2020), aún no se tiene un conocimiento robusto de su distribución actual ni de su hábitat, entre otros. De hecho, actualmente esta chinchilla está considerada "En Peligro Crítico" en la mayoría de los países con presencia histórica, así como también en Chile que ha sido clasificada con la misma categoría por el Reglamento de Conservación de Especies (RCE; D.S. N ${ }^{\circ}$ 29/2011 del Ministerio del Medio Ambiente). Dado el estado de sus poblaciones, la chinchilla andina, se ha considerado como monumento nacional en Chile (D.S. Nº2/2006 del Ministerio de Agricultura). Además, muy recientemente se ha creado un Plan de Recuperación, Conservación y Gestión (RECOGE; D.S. $N^{\circ}$ 19/2020 del Ministerio del Medio Ambiente), para mejorar su categoría de conservación y disminuir su estado de amenaza para los próximos diez años.

En el presente trabajo se describe un nuevo registro de chinchilla andina entre el Salar de Tara y el de Aguas Calientes I de la cordillera de la Región de Antofagasta, en la comuna de San Pedro de Atacama. Dicho registro fue realizado mediante cámaras trampa en un estudio de diversidad de especies y, en él, se detalla el sitio y se caracteriza el hábitat con el fin de aportar nuevos datos para la especie.

\section{ÁREA DE ESTUDIO Y METODOLOGÍA}

Se diseñó un estudio mediante cámaras trampa de la diversidad de meso y macrofauna en la comuna de San Pedro de Atacama, Chile. Concretamente en el sector 1, el Salar de Tara y Aguas Calientes I, de la Reserva Nacional Los Flamencos (RNLF), una de las áreas silvestres protegidas (ASP) de CONAF. Este sector con una extensión total de 36674 ha y ubicado a una altura promedio de $4300 \mathrm{msnm}$, está conformado principalmente por los dos grandes cuerpos de agua que le dan su nombre (CONAF 2008). Además, el Salar de Tara forma parte del listado de Humedales de Importancia Internacional de la Convención de Ramsar para proteger y preservar, entre otros, el hábitat de las aves acuáticas presentes en la zona.

El diseño experimental consta de 34 celdas, en la extensión de todo el sector 1 del Salar de Tara y Aguas Calientes I (Ahumada et al. 2011; Shannon et al. 2014). Las celdas muestrales son de $1 \mathrm{~km}^{2}$ (Hedwig et al. 2018), separadas entre sí por una distancia de 1,5 km (Tobler et al. 2008; Kinnaird y O’Brien 2012) y se dispusieron de manera aleatoria y estratificada en relación al tipo de biotopo. Las cámaras trampa se ubicaron a una distancia máxima de $250 \mathrm{~m}$ del punto central de cada una de las celdas, en aquellos lugares donde se encontraron rastros de fauna, para así, maximizar la probabilidad de captura (Cusack et al. 2015; 
Colyn et al. 2018). Por el contrario, si no se encontraba ningún rastro de fauna, la cámara se instalaba en el mismo punto central (Wearn y Glover-Kapfer 2017). Estas se ataron a rocas o estacas a una altura de $20 \mathrm{~cm}$ del suelo sin la utilización de ningún tipo de atrayente, se configuraron para mantenerse activas durante las 24 horas del día y registrar tres fotografías en ráfaga al detectar movimiento cada 30 segundos. Asimismo, las cámaras se dejaban por un periodo de 34 días en terreno para luego retirarlas y volver a instalarlas de la misma manera en la siguiente estación del año, obteniendo así los datos anuales de 2020 organizados por las cuatro estaciones.

\section{RESULTADOS}

Se alcanzó un total de 3509 registros independientes y 4624 cámaras/noche de esfuerzo muestral. Sin embargo, solo en una de las 34 cámaras trampa empleadas se registró la chinchilla andina (Chinchilla chinchilla). El registro se efectuó de noche en la campaña de primavera (noviembre 2020) y constó de la presencia de dos series de fotografías distintas, separadas por más de 50 horas la una de la otra (Figura 1). Ambas series, mostraban lo necesario para la determinación de la especie: cuerpo de unos $34 \mathrm{~cm}$, pelaje de tonos gris y sedoso, orejas grandes y redondas, y cola curva y muy peluda (Iriarte et al. 2011).

La especie se encontró dentro del sector $1\left(23^{\circ} 02^{\prime} 17^{\prime \prime} \mathrm{S}-67^{\circ} 21^{\prime} 41^{\prime \prime} \mathrm{O}\right)$ entre medio de las dos lagunas (Figura 2) y, además, bajo la protección del sitio Ramsar. El lugar formaba parte de una vasta planicie de rocas pequeñas a $4400 \mathrm{msnm}$ y con la presencia de grandes rocas amontonadas, repletas de cavidades, formando pequeños roqueríos aislados y solitarios (Figura 3). Por otro lado, la vegetación colindante que se identificó se constituía de Parastrephia quadrangularis (Familia Asteraceae), Festuca chrysophylla (Familia Poaceae), Festuca orthophylla (Familia Poaceae), Deyeuxia sp (Familia Poaceae) y Mulinum crassifolium (Familia Apiaceae), todas ellas con escasa presencia en el área y siendo las especies de Festuca las más abundantes. Además, entre las rocas dentro de pequeñas cuevas, se encontraron varios defecaderos de chinchilla andina. Referente a la presencia de agua en el lugar, el cuerpo de agua dulce más próximo a

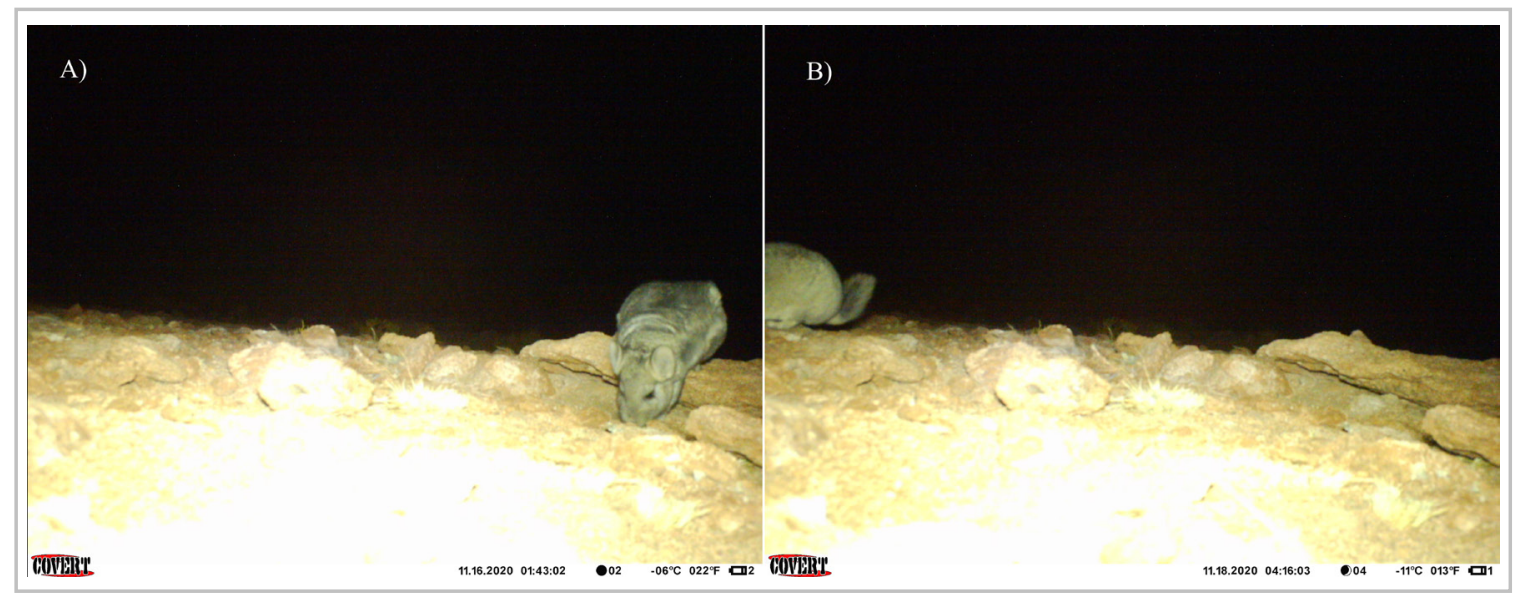

Figura 1. A) Fotografía del primer registro de chinchilla andina en la RNLF; B) Fotografía del segundo registro de chinchilla andina en la RNLF. 


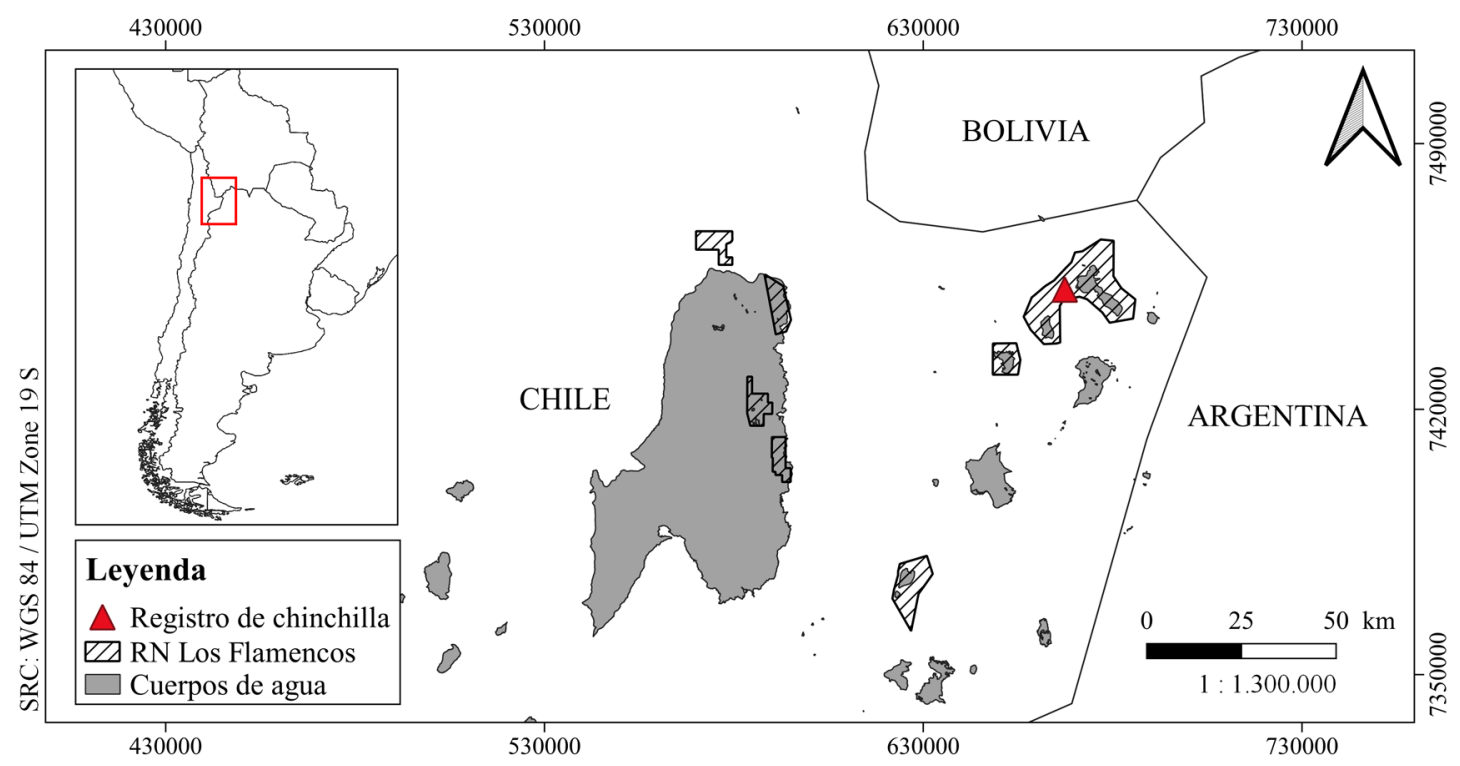

Figura 2. Mapa de la localización de la nueva colonia de chinchilla andina en el sector 1 de la RNLF.

dicho punto se encuentra a una distancia de unos $2500 \mathrm{~m}$, muy parecido a lo observado en Navarro et al. (2019). En consecuencia, parece que los lugares sin agua no deberían ser un problema para la especie, como también se detalla para las colonias estudiadas en la REA (Delgado 2020). Por otro lado, en ese mismo sitio, a lo largo del año se registraron también otras especies objetivo del estudio de diversidad como: la vicuña austral (Vicugna vicugna vicugna) y la perdiz de la puna (Tinamotis pentlandii). Sin embargo, la abundancia de registros de fauna presentes en dicho lugar es pobre, representando solamente un $0,37 \%$ del total obtenido al final del estudio.

\section{DISCUSIÓN}

Este registro en el sector 1 de la RNLF es de gran importancia, ya que su presencia no estaba descrita para esta zona. Con él, se pretende aportar información actualizada de la especie y de su hábitat, extendiendo así su distribución geográfica actual hacia el norte. En consecuencia, se espera que estos resultados sirvan de utilidad para el nuevo plan RECOGE de la chinchilla, al igual que para los futuros modelos de distribución potencial que se generen para dicha especie.

Asimismo, este nuevo hallazgo subraya la importancia del estudio de la fauna y de su relevamiento en las ASP, así como de la función que tienen estas en la conservación y protección de los ecosistemas nativos, que son capaces de refugiar especies tan frágiles e icónicas como la desaparecida chinchilla andina. Por consiguiente, este trabajo respalda el cierre total preventivo que se hizo del sector (RES. No 117/2019), prohibiéndose el ingreso de todo tipo de visitantes, en pos del estudio y de la protección del ecosistema para su cuidado y bienestar. A raíz de lo presente, se recomienda proseguir con el estudio de diversidad y, en especial, con el monitoreo de la chinchilla en el sector, así como también replicarlo a otras ASP del país con el objetivo de encontrar nuevos sitios de presencia. Además, se cree importante estudiar también la estructura genética de las colonias que se van encontrando, para poder determinar el flujo genético entre las poblaciones, y reunir la información necesaria para examinar la sustentabilidad global de la especie. 


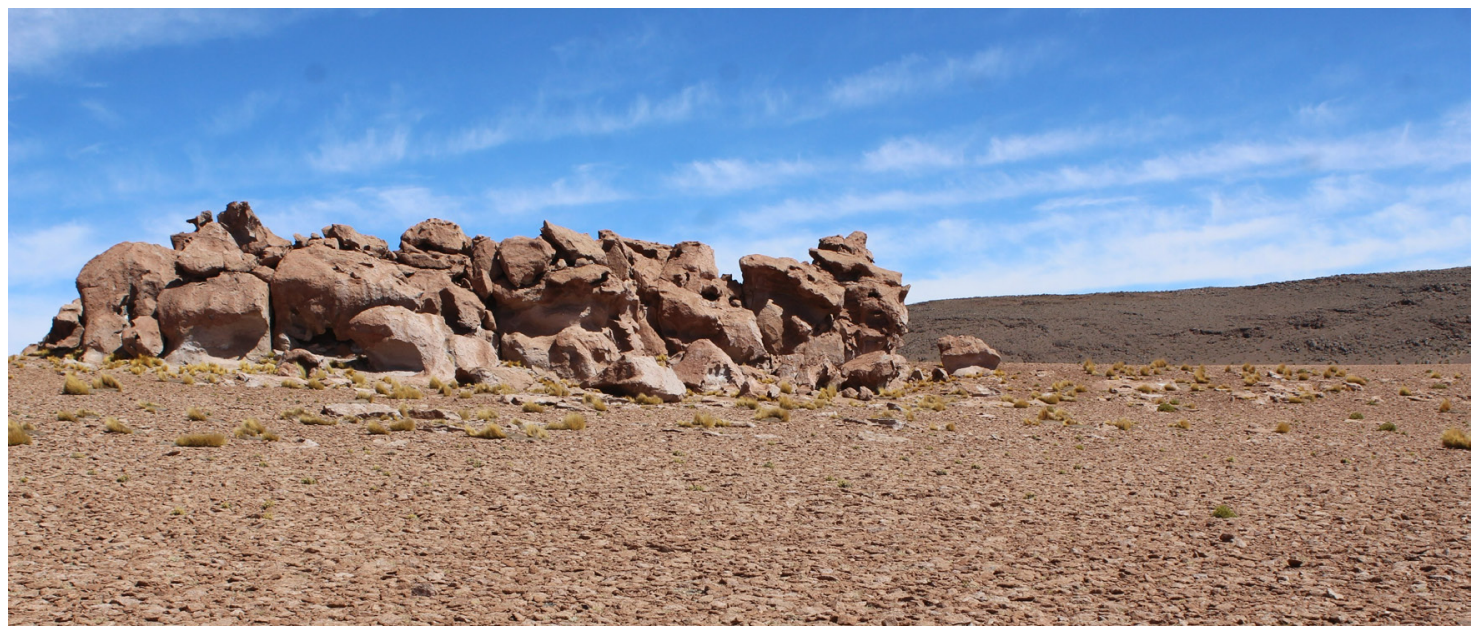

Figura 3. Lugar y tipo de hábitat donde se registró la colonia de chinchilla andina en la RNLF.

Ya para finalizar, puesto que la nueva colonia encontrada de chinchilla andina se ubica en un trifinio entre los países de Chile, Bolivia y Argentina, se sugiere a este último que se plantee la posibilidad de estudiar la presencia del elusivo roedor cerca de este punto, en la Provincia de Jujuy. Ya que tanto Bolivia como Chile comparten la presencia de esta especie en dicha localización. Asimismo, se recomienda incluir en los actuales modelos de distribución potencial, los datos de hábitat de este nuevo registro, con el fin de conocer mejor a la especie y de ayudar en su protección.

\section{AGRADECIMIENTOS}

Queremos agradecer a todos los de CONAF y, en específico, al equipo de la RNLF que han apoyado y han aportado en la realización del estudio de diversidad de fauna del sector de Tara y Aguas Calientes I, así como en el redescubrimiento de la chinchilla andina en dicha área. Además, reconocer la ayuda brindada por la Comunidad Indígena Atacameña de Toconao en los inicios de este estudio, al colaborar en la instalación de las cámaras trampa. Igualmente, le damos las gracias a Diego Aramayo por echarnos una mano con la identificación de las especies de flora que constituían el hábitat de la chinchilla.

\section{REFERENCIAS BIBLIOGRÁFICAS}

AHUMADA, J. A., C.E.F. SILVA, K. GAJAPERSAD, C. HALlAM, J. HURTADO, E. MARTIN, A. MCWILLIAM, B. MUGERWA, T. O'BRIEN, F. ROVERO, D. SHEIL, W.R. SPIRONELLO, N. WINARNI y S.J. ANDELMAN. 2011. Community structure and diversity of tropical forest mammals: Data from a global camera trap network. Philosophical Transactions of the Royal Society B: Biological Sciences, 366(1578): 2703-2711.

COLYN, R. B., F.G.T RADLOFF y M.J. O'RIAIN. 2018. Camera trapping mammals in the scrubland's of the Cape Floristic Kingdom - The importance of effort, spacing and trap placement. Biodiversity and Conservation, 27(2): 503520 .

CORPORACIÓN NACIONAL FORESTAL. 2008. Actualización Plan de Manejo Participativo Reserva Nacional Los Flamencos. Antofagasta, Chile. (p. 143).

COPA, M. E., G.S. MENDIETA y A.L. DEANE. 2014. Distribución potencial de la chinchilla de cola corta (Chinchilla chinchilla, Rodentia) en el sudoeste de Bolivia. Ecología en Bolivia, 49(2): 65-76.

CUSACK, J. J., A. J. DICKMAN, J. M. ROWCLIFFE, C. CARBONE, D.W. MACDONALD y T. COULSON. 2015. Random versus Game Trail-Based Camera Trap Placement Strategy for Monitoring Terrestrial Mammal Communities. 
PLOS ONE, 10(5), e0126373. https://doi.org/10.1371/journal.pone.0126373

DEANE, A. 2020. Potential Distributions of Chinchilla chinchilla in Chile. Zenodo. https://doi.org/10.5281/ ZENODO.4269844

DELGADO, E. 2020. Historia natural de la chinchilla de cola corta (Chinchilla chinchilla) en la Reserva de Fauna Andina Eduardo Avaroa, Bolivia. Ecología en Bolivia, 55(1): 62-65.

DELGADO, E., L.F. PACHECO, J. SALAZAR-BRAVO y O. ROCHA. 2018. La chinchilla de cola corta (Chinchilla chinchilla) en Bolivia: Comentarios sobre localidades reportadas y bases para su conservación. Ecología en Bolivia, 53(1): 31-38.

HEDWIG, D., I. KIENAST, M. BONNET, B.K. CURRAN, A. COURAGE, C. BOESCH, H.S. KÜHL y T. KING. 2018. A camera trap assessment of the forest mammal community within the transitional savannah-forest mosaic of the Batéké Plateau National Park, Gabon. African Journal of Ecology, 56(4): 777-790.

IRIARTE, A., N. LAGOS y R. VILLALOBOS. 2011. Los Mamíferos de la Región de Antofagasta. Ediciones Flora \& Fauna Chile.

JIMÉNEZ, J. E. 1996. The extirpation and current status of wild chinchillas Chinchilla lanigera and C. brevicaudata. Biological Conservation, 77(1): 1-6.

KINNAIRD, M. F. y T.G. O'BRIEN. 2012. Effects of Private-Land Use, Livestock Management, and Human Tolerance on Diversity, Distribution, and Abundance of Large African Mammals: Livestock and Large Mammals in Kenya. Conservation Biology, 26(6): 1026-1039.

LAGOS, N., R. VILLALOBOS y A. IRIARTE. 2012. Nuevos registros de poblaciones de chinchilla de cola corta, Chinchilla chinchilla (Rodentia, Chinchillidae) en la cordillera de la Región de Atacama. Boletín del Museo de Historia Natural (Chile), 61: 191-196.

MELLA, J., J.A. SIMONETTI, A.E. SPOTORNO y L.C. CONTRERAS. 2002. Mamíferos de Chile. En G. Ceballos y J. A. Simonetti (eds). Diversidad y Conservación de los Mamíferos Neotropicales (p. 151-183). CONABIO-UNAM.

NAVARRO, S. M., P.P. BARAHONA y J.M. ÁVILA. 2019. Análisis de nuevos registros de la chinchilla de cola corta (Chinchilla chinchilla, Lichtenstein, 1829) en la Región de Atacama, Chile. Boletín del Museo Nacional de Historia Natural, 68(1-2): 57-71.

ORTIZ, P. E., F.R. GONZÁLEZ, J.P. JAYAT, U.F.J. PARDIÑAS, S. CIRIGNOLI y P. TETA. 2010. Dieta del búho magallánico (Bubo magellanicus) en Los Andes del Noroeste Argentino. Ornitologia Neotropical, 21: 591-598.

PACHECO, V., S. DIAZ, L. GRAHAM-ANGELES, M. FLORES-QUISPE, G. CALIZAYA-MAMANI, D. RUELAS y P. SÁNCHEZ-VENDIZÚ. 2021. Lista actualizada de la diversidad de los mamíferos del Perú y una propuesta para su actualización. Revista peruana de biología, 28(4): e21019. http://dx.doi.org/10.15381/rpb.v28i4.21019

RIQUELME, J., J. VEGA, C. CONTRERAS y N. AMADO. 2015. Nuevos registros de chinchilla (Chinchilla chinchilla) para el Parque Nacional Llullaillaco, Región de Antofagasta. Biodiversidata, 3: 94-96.

RUDOLPH, W. E. 1955. Licancabur: Mountain of the Atacamenos. Geographical Review, 45(2): 151-171.

SALINAS, H., N. REBOLLEDO y G. LOBOS. 2019. Nuevo Registro y Ampliación del Rango Altitudinal de la Chinchilla de Cola Corta (Chinchilla chinchilla) en el Norte de Chile. La Chiricoca, 24: 15-21.

SHANNON, G., J.S. LEWIS y B.D. GERBER. 2014. Recommended survey designs for occupancy modelling using motion-activated cameras: Insights from empirical wildlife data. PeerJ, 2, e532. https://doi.org/10.7717/peerj.532.

SPOTORNO, A.E., J.P. VALLADARES, J.C. MARIN, R.E. PALMA y C. ZULETA. 2004. Molecular divergence and phylogenetic relationships of chinchillids (Rodentia: Chinchillidae). Journal of Mammalogy, 85(3): 384-388.

TOBler, M. W., S.E. CARRILlO-PERCASTEGUI, R. LEITE PITMAN, R. MARES y G. POWELL. 2008. An evaluation of camera traps for inventorying large- and medium-sized terrestrial rainforest mammals. Animal Conservation, 11(3): 169-178.

VALLADARES, P., M. ESPINOSA-MOLINA, M. TORRES, E. DIAZ, N. ZELLER, J. de LA RIVA, M. GRIMBERG y Á. SPOTORNO. 2012. Nuevo registro de Chinchilla chinchilla (Rodentia, Chinchillidae) para la Región de Atacama, Chile. Implicancias para su estado de conservación. Mastozoologia Neotropical, 19(1): 173-178.

VALlADARES, P., C. ZULETA y Á. SPOTORNO. 2014a. Chinchilla lanigera (Molina 1782) and C. chinchilla (Lichtenstein 1830): Review of their distribution and new findings. Animal Biodiversity and Conservation, 37(1): 8993. 
VALLADARES, P., Á. SPOTORNO y C. ZULETA. 2014b. Natural history of the Chinchilla genus (Bennett 1829): Considerations of their ecology, taxonomy and conservation status. Gayana, 78(2): 135-143.

VALLADARES, P., Á. SPOTORNO, A. CORTES y C. ZULETA. 2018. Chinchilla chinchilla (Rodentia: Chinchillidae). Mammalian Species, 50(960): 51-58.

WEARN, O. R. y P. GLOVER-KAPFER. 2017. Camera-trapping for conservation: a guide to best-practices. WWF Conservation Technology Series, WWF-UK, Working, United Kingdom.

Recibido: 27/sep/2021; Aceptado: 03/dic/2021; Administrado por Jhoann Canto H. 